\title{
Characterization and Simulation of a Magnetized Sample
}

\author{
Michael Linnert', Reinhard Lerch ${ }^{1}$, Alexander Sutor ${ }^{1}$, Stefan J. Rupitsch ${ }^{1}$ \\ ${ }^{1}$ Chair of Sensor Technology, University Erlangen-Nuremberg (FAU), Paul-Gordan-Str. 3/5, 91052 \\ michael.linnert@fau.de
}

\begin{abstract}
For several decades, magnetic sensors have been exploiting the nonlinear relation between the magnetic field strength and the magnetic flux density. Whereas strong saturation effects were most relevant in the past, today energy consumption of modern sensors plays an increasingly important role. Recent semihard magnetic materials concern this issue by retaining a significant magnetic flux density even when all excitation currents are turned off. Nevertheless, in contrast to classical hard magnets, it is relatively easy to commutate them. With sensor development cycles becoming shorter, there is an increasing demand for accurate numerical models concerning hysteresis. In this contribution, we present a new combination of scalar and vector material characterization techniques to compute the remanent magnetic field of a magnetized sample by static finite element (FE) simulations. Vacuumschmelze offers a suitable semihard magnetic material with a coecivity field around $2 \mathrm{kA} / \mathrm{m}$ that is available as thin stripes. In a first step, the Sensorvac material will be characterized to obtain meaningful material properties required for simulations. With material parameters having been identified, the remanent magnetic field of the magnetized sample will be computed. As it is revealed, simulation results coincide very well with laboratory measurements. In future, the model can be applied to design complex sensors.
\end{abstract}

Key words: FEM, magnetics, hysteresis, material properties, remanent field

\section{Introduction}

According to Coey [7], magnetic materials underwent an immense development during the 20th century. Today, with realizable coercivity field strengths from $10^{-1}$ to $10^{7} \mathrm{~A} / \mathrm{m}$, there are many materials for all kinds of applications. Even the shape of hysteresis is almost variable by choose of a proper material. These materials span a wide field of applications ranging from data storage over telecommunications to consumer electronics and sensors. With a market of about 30 b\$ per year [7] and their high flexibility, it is worth to model these nonlinear materials to study their applicability in modern sensors.

One of the first sensors exploiting the nonlinear saturation between the magnetic field strength and the flux density was the fluxgate magnetometer invented by Friedrich Förster in 1937 [8]. The saturation effect allows to measure magnetic fields very precisely. In contrast to the simple saturation within the fluxgate magnetometer, modern sensors even use the hysteresis itself. In department stores, magnetic stripes are attached to products to secure them against theft [2]. When a customer leaves the store through a gate, which provides an alternating magnetic field, higher harmonics will be induced in the response signal due to saturation effects within the magnetic stripes. These harmonics will vanish if the label is deactivated by magnetization at the point of sale as the stripe retains a certain flux density. Nowadays, these materials can also concern energy saving issues, wherever an electromagnet should maintain an alternating d.c. field for a long period.

The aim of this work is to develop a static FE model for the computation of the hysteretic involved remanent field of a magnetized sample (Fig. 1) when there are no more excitation currents. Nevertheless, the same approach would also hold for more simple nonlinear saturation effects.

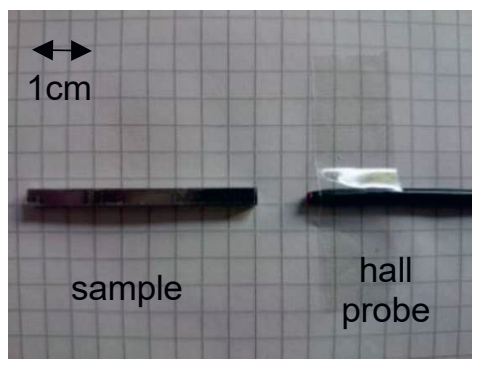

Fig. 1. Stacked semihard Sensorvac sample on the left, hall probe on the right.

At first, we will study the analytical basics for a magnetic dipole. Secondly, we will explain the measurement setups used for the characterization of magnetic materials. This will be followed by the derivation of a FE model for the simulation 
of the demagnetization process within the sample. Finally, simulation results will be validated by laboratory measurements.

\section{Theory}

Figure 2 sketches a magnetic dipole, whose remanent field after magnetization will be computed.

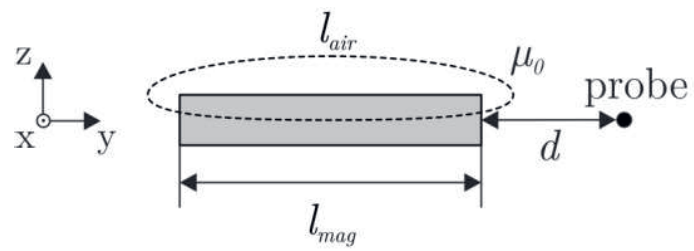

Fig. 2. Geometric model of a magnetic sample.

This can be interpreted as a special case of an open magnetic circuit. With Ampère's law

$$
H_{\text {mag }} l_{\text {mag }}+H_{\text {air }} l_{\text {air }}=0
$$

and magnetic flux conservation

$$
B_{\text {air }} A_{\text {air }}=\kappa B_{\text {mag }} A_{\text {mag }}
$$

a condition for the flux density within the material $B_{\text {mag }}$ can be derived with respect to the geometric properties (cross-section $A$, length $l$ ) and the field strength $H_{m a g}$

$$
B_{m a g}=-\frac{\mu_{0}}{\kappa} \frac{A_{\text {air }}}{A_{\text {mag }}} \frac{l_{\text {mag }}}{l_{\text {air }}} H_{m a g} .
$$

The index mag denotes the magnetic material, the index air the surrounding air, $\kappa<1$ is the stray field factor and $\mu_{0}$ is the permeability of vacuum, respectively. Together with the hysteretic behavior of the material

$$
B_{m a g}=B_{m a g}\left(H_{m a g}\right),
$$

a nonlinear equation system is formed, whose solution determines the flux density as well as the field strength within the material. This can also be interpreted geometrically (Fig. 3) as the intersection of the geometric-depended linear function Eq. (3) with the material-depended hysteretis loop Eq. (4).

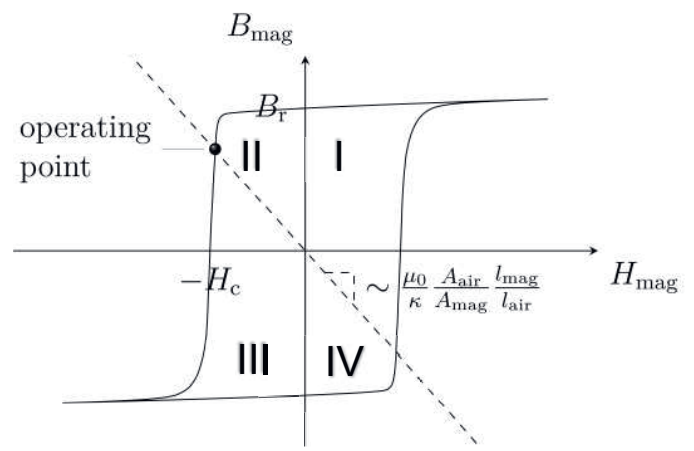

Fig. 3. Geometric determination of the operating point as the intersection of a geometric-depended linear function with the material-depended hysteresis.

\section{Epstein Frame}

To solve for the magnetic field, the material behavior should be characterized first. With the Epstein frame [1], a standardized measurement device of high repeatability and reliability is available.

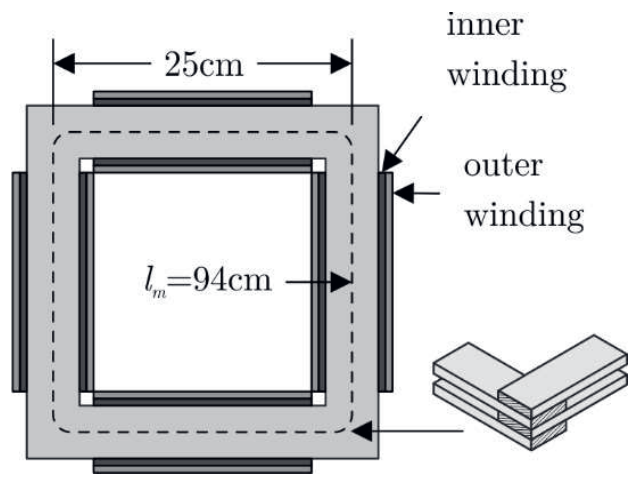

Fig. 4. Epstein frame for material characterization. Sample material stacked inside the coils.

As shown in Fig. 4, the Epstein frame consists of a set of four coils. Together, the outer and inner windings form a transformer. With the outer windings being connected in series, the magnetic field strength $H_{\text {mag }}$ is known from the excitation current $I_{1}$, the number of windings $N$, and the effective path length $l_{m}$

$$
H_{m a g}=\frac{N I_{1}}{l_{m}} .
$$

Due to the smooth field in the corners, the effective path length is often set to $0.94 \mathrm{~m}$ [3] but can also be calibrated by a material with known coercivity field. Finally, the induced voltage in the inner windings $U_{2}$ can be used to obtain the flux density within the sample

$$
B_{\text {mag }}=-\frac{1}{N A} \int U_{2} d t,
$$

where $A$ is the cross-section of the coils and the inner windings have been connected in series. Of course, the flux density can also be calibrated by a material with known remanence. The stacked sample material inside the coils influences the coupling of the coils in a specific way (Fig. 5).

Although the Epstein frame is often used, it suffers from some disadvantages. First, numerical integration is difficult because of unknown initial states. This requires a vertical centering of the measured hysteresis loop and that is why the Epstein frame is only suitable for materials with symmetric loops. Moreover, the air flux between the stacked samples must be compensated by hardware or software. Furthermore, as the Epstein frame integrates the field strength along its length it can only be used for the characterization of isotropic materials. 


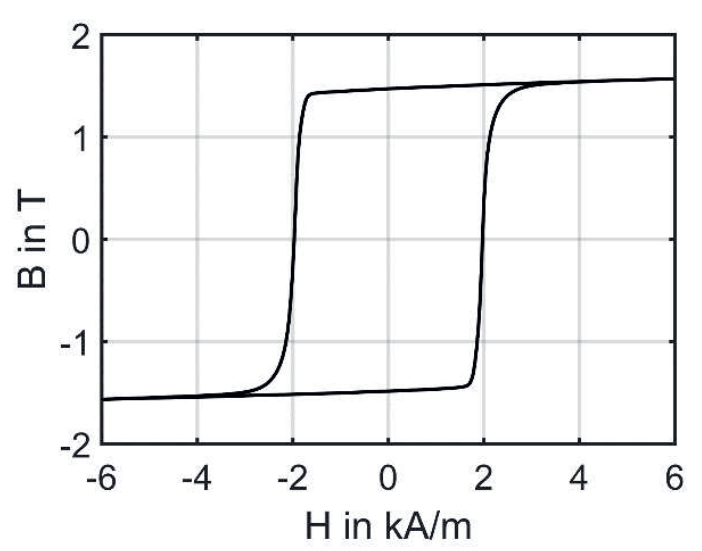

Fig. 5. Hysteresis loop measured by the Epstein frame

\section{Vibrating Sample Magnetometer (VSM)}

Whereas the Epstein frame only characterizes the average flux density within a sample, the vibrating sample magnetometer VSM also allows a vector characterization of anisotropic materials [9].

A rod placed between two electromagnets, which are used to generate a static magnetic field, holds the sample (Fig. 6). Now, the rod is excited to vibrate mechanically at a specific amplitude and frequency.

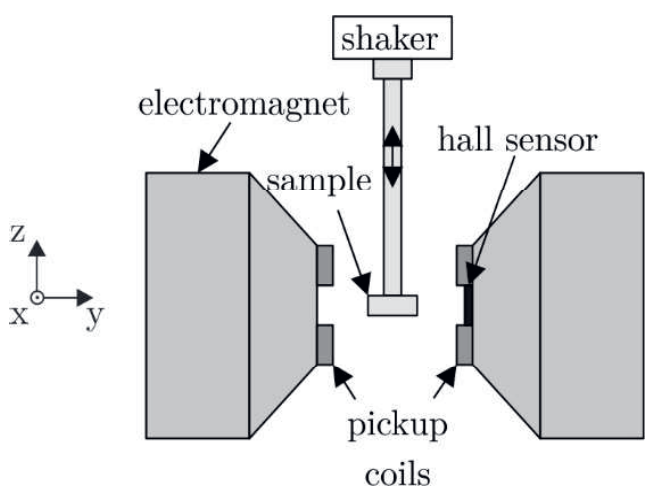

Fig. 6. Vibrating sample magnetometer for material characterization.

If the sample is considered as a vibrating dipole, a voltage $u(t)$ is induced in the pickup coils

$$
u(t)=m G(z) z_{0} \omega \cos \omega t,
$$

where $m$ is the magnetic moment, $G(z)$ is a geometric factor, $\omega$ and $z_{0}$ are the frequency and the amplitude of the shaker, respectively. Equation (7) assumes a sinusoidal excitation. Owing to the small sample size, a lock-in amplifier is used to amplify the induced voltage. The amplifier's output, the root mean square value $U_{r m s}=K m$ of the induced voltage, is then proportional to $m$. The calibration factor $K$ can be determined by a sample with a known magnetic moment. For a uniformly magnetized sample, we finally obtain the magnetic flux density within a sample of volume $V$

$$
B_{m a g}=\mu_{0}\left(H_{m a g}+\frac{U_{r m s}}{K V}\right)
$$

The field strength $H_{\text {mag }}$ is measured by a small hall sensor placed between the pickup coils.

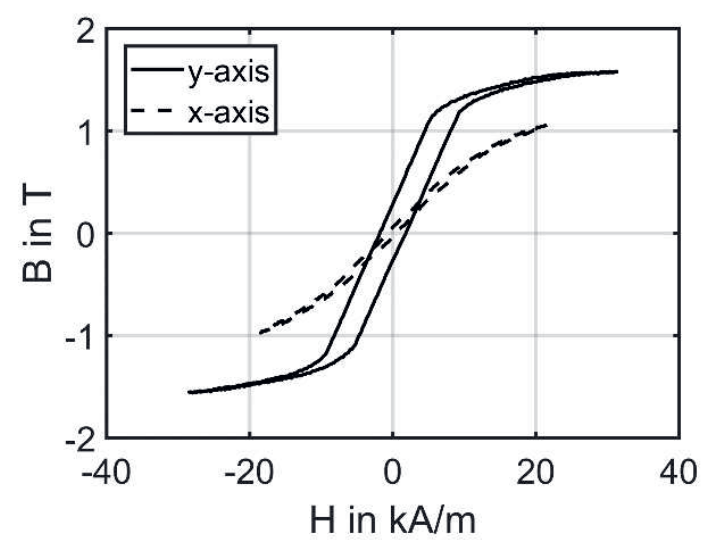

Fig. 7. Hysteresis loops measured by the vibrating sample magnetometer for different directions. Predominant direction solid.

Figure 7 shows the highly anisotropic behavior of the Sensorvac material. There is mainly hysteresis only in one predominant direction (y-axis). This direction is identical to the mechanical direction of rolling of the Sensorvac stripes.

As the sample is magnetized in an open magnetic circuit, demagnetization fields can no more be neglected. Since the sample is magnetized by an applied external field, the internal field counteracts the induced magnetization. It can be shown mathematically that the demagnetization field can only be neglected for disk-shaped samples with a diameter much larger than its thickness [4]. This limits the VSM to the characterization of materials being available as small disks or applications having the same small geometric dimensions as the sample used for the VSM.

If the hysteresis curve measured by the Epstein frame (Fig. 5) is compared with those recorded by the VSM (Fig. 7), one can clearly see demagnetization effects if it is considered that both have been measured for the same Sensorvac material. Otherwise, the hysteresis curve in predominant direction (Fig. 7), measured with the VSM, would have matched the Epstein measurement. Demagnetization effects owing to the rectangular shape of the Sensorvac stripes are too strong. If the sample would be shorter, demagnetization effects even would increase. Since the magnet, which should be simulated, is significantly longer than the sample used for the VSM, we will prefer the Epstein measurement for the hysteretic part of the simulation. This is also tol- 
erable because the Sensorvac stripes show hysteresis mainly in rolling direction. The VSM measurements were used to obtain the constant permeability for the remaining directions (constant slope of dashed line in Fig. 7).

\section{FE Modeling}

Having characterized the sample material, we are now able to develop a FE model. For the magnetostatic problem of the computation of the remanent field, there are no more currents, i.e. $\nabla \times \mathrm{H}=0$. This implies that we can define a magnetic scalar potential $V_{m}$ similar to the static electric potential [5]

$$
H=-\nabla V_{m}
$$

Combining the constitutive relation $B=\mu_{0}(H+M)$ with the solenoidality of the magnetic field $\nabla B=0$, we obtain Poisson's equation for magnetostatic problems without electric currents

$$
-\nabla \mu_{0}\left(\nabla V_{m}-M\right)=0
$$

that can be solved by FE solvers to compute the magnetic field. Since the magnetization $\mathrm{M}=-\left(\mu_{\mathrm{r}}-1\right) \nabla V_{m}$ itself depends on the relative permeability, we must provide the permeability tensor $\mu_{\mathrm{r}}$ for the magnetic sample

$$
\mu_{r}=\left[\begin{array}{ccc}
\mu_{r, x x} & 0 & 0 \\
0 & B_{y}\left(H_{y}\right) /\left(\mu_{0} H_{y}\right) & 0 \\
0 & 0 & 1
\end{array}\right] .
$$

The permeability tensor models hysteresis only in y-direction because the VSM measurements showed that the sample material exhibits one predominant magnetization direction in rolling direction (Fig. 7). This is done by interpolating the already presented demagnetization curve measured with the Epstein frame (second quadrant of Fig. 3). That approach is permissible because all physical operating points lie in the second or fourth quadrant of the hysteresis loop. In the other directions, the material is supposed to behave linearly; that was validated with the VSM. The permeability value in $\mathrm{x}$-direction is constant and can be derived from the constant slope of the dashed line in Fig. 7. Being stacked, the material exhibits the permeability of air in z-direction, as the magnetic flux is not able to couple from one stripe to its neighbor due to thin air layers between the stacked stripes. Moreover, no cross interactions between the individual directions are assumed. It should be noted that the model is not limited to geometries with the predominant direction being parallel to one of the coordinate axes. Geometries that are more complex can be modeled by curvilinear coordinate systems [6]. The deformed coordinate system can be computed previously by a pseudo flow computation within the sample. This requires an inlet and outlet to be defined in predominant direction.

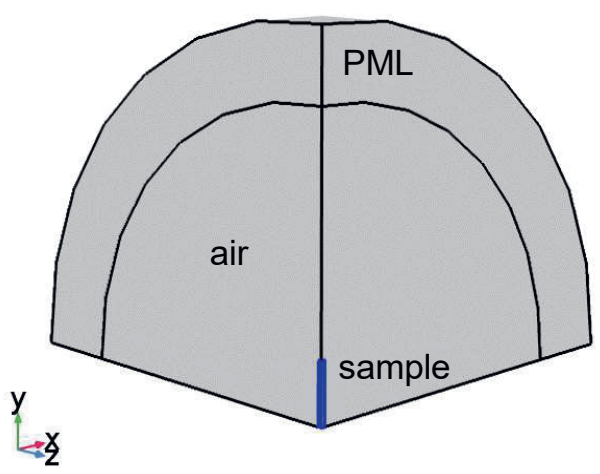

Fig. 8. FE-Model of a magnetic sample under utilization of symmetries. PML and air domain included.

In order to prevent the trivial solution $H=0 \mathrm{~A} / \mathrm{m}$ the initial gradient of the magnetic scalar potential within the sample must differ from zero. To reduce the numerical effort, all symmetries of the sample have been exploited (Fig. 8). Moreover, a perfectly matched layer (PML) with a high damping of the field was used to reduce the necessary size of the surrounding air.

\section{FE Results}

Figure 9 shows the computed magnetic flux density around the sample. The surface plot is logarithmic scaled. Of course, this is the expected field of a bar magnet.

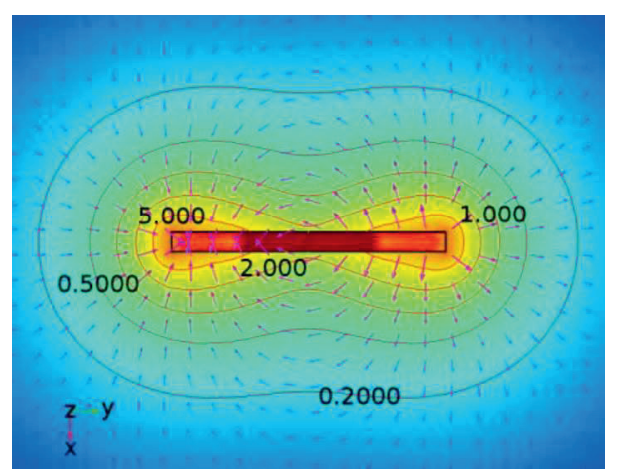

Fig. 9. Magnetic flux density $B$ of the magnetized sample. Isolines in $m T$.

The computed operating point in the middle of the magnet is depicted in Fig. 10. As it was expected, it lies on the measured demagnetization curve. The very low operating point is not surprising because the bar magnet forms an open magnetic circuit, Eq. (3). 


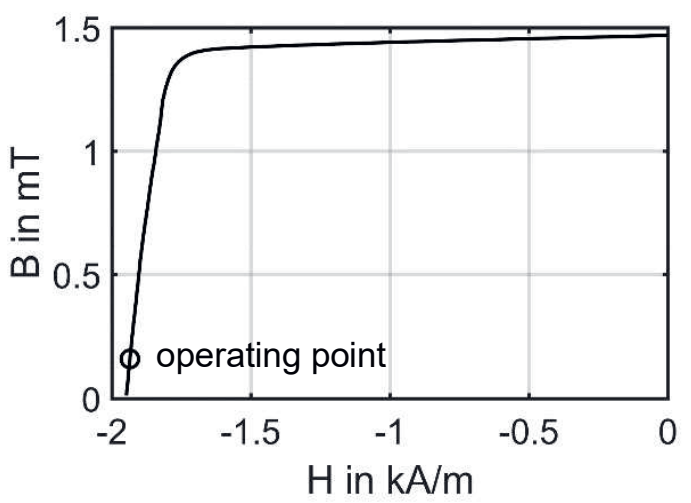

Fig. 10. Demagnetization curve with computed operating point.

In Fig. 11 a comparison between the computed and the measured flux density on the axis of the sample is shown. Before measurement, the sample was excited into saturation. For this reason, we were able to use the previous characterized major loop for the simulation. Indeed, a simulation on smaller minor loops is also possible, but it would be much harder to validate the simulation, because in contrast to the simulation we do not know on which loop the material was excited in reality. Due to the good agreement between simulation results and measurements, both the FE-model as well as the previous material characterization is validated.

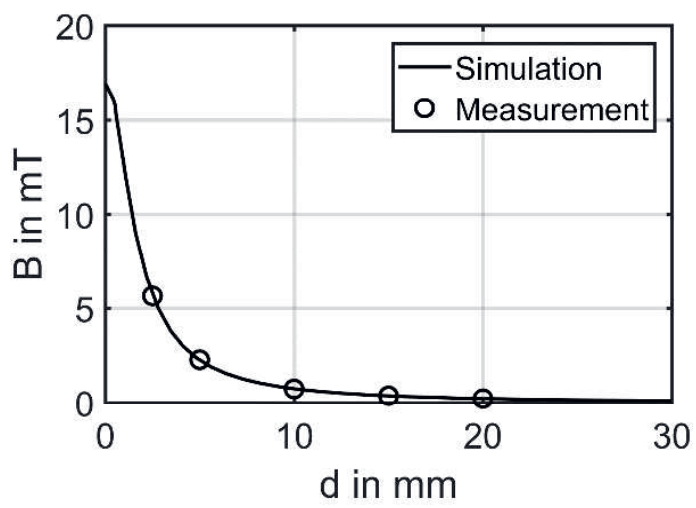

Fig. 11. Comparison between simulation and measurement. Flux density on the axis of the dipole for different distances to the sample.

\section{Conclusion}

This work shows how to model magnetic materials. We presented a practicable workflow to obtain a reasonable FE model. Priority was set on the task of material characterization. Our new approach combines the measured demagnetization curve with the vector characterization of the sample material. This also allows using the demagnetization curve directly in an intuitive way. Whereas the Epstein frame measurements model the hysteretic part of the simulation in rolling direction of the Sensorvac stripes, the Vibrating Sample Magnetometer characterizes the linear material behavior in the other directions. It was demonstrated, that strong demagnetization effects within the sample prohibit modeling the hysteretic part by VSM measurements.

Starting from Maxwell's equations a static finite element model was developed to simulate the remanent field of the magnetized sample. The computed field shows the characteristics of the expected bar magnet. As it was presented, simulation results coincide very well with laboratory measurements. The model is now ready for sensor development of geometries that are too complex and costly to construct as prototypes.

\section{References}

[1] S. Tumanski, Handbook of magnetic measurements, CRC Press (2016); doi: 10.1201/b10979

[2] G. Herzer, Sensorphysik: Der große Lauschangriff auf Ladendiebe: Waren lassen sich durch weichmagnetische Sensorstreifen oder elektrische Schwingkreise vor Diebstahl sichern, Physikalische Blätter, 57. Jg., Nr. 5, 43-48 (2001)

[3] P. Marketos, S. Zurek and A. J. Moses, A Method for Defining the Mean Path Length of the Epstein Frame, IEEE Transactions on Magnetics, vol. 43, no. $6,2755-2757$ (2007); doi: 10.1109/TMAG.2007.894124

[4] S. Bi, Characterization and Modeling of Hysteresis in Ferrormagnetic Material (2014), PHD-Thesis

[5] J. Simkin, C.W. Trowbridge, On the use of the total scalar potential on the numerical solution of fields problems in electromagnetics, International journal for numerical methods in engineering, 14. Jg., Nr. 3, 423-440 (1979); doi: 10.1002/nme.1620140308

[6] N. Bannach, Defining Curvilinear Coordinates for Anisotropic Materials, Comsol Blog (2014); https://www.comsol.com/blogs/defining-curvilinear-coordinates-anisotropic-materials/

[7] J. Coey, Magnetic materials, Journal of Alloys and Compounds 326.1 2-6 (2001); doi: 10.1016/S0925-8388(01)01239-7

[8] P. Ripka, Advances in fluxgate sensors, Sensors and Actuators A: Physical, 106. Jg., Nr. 1, 8-14 (2003); doi: 10.1016/S0924-4247(03)00094-3

[9] A. Sutor, J. Kallwies, R. Lerch, Vektorielles messen und modellieren von magnetischer hysterese, tm-Technisches Messen Plattform für Methoden, Systeme und Anwendungen der Messtechnik 79.4, 220-228 (2012); doi: 10.1524/teme.2012.0225 